\title{
One-year periodization of training loads of Russian and Norwegian elite cross-country skiers
}

\author{
EVGENY B. MYAKINCHENKO $\longleftarrow$, ANDREY S. KRIUCHKOV, NIKITA V. ADODIN, MARINA A. DIKUNETS, \\ MIKHAIL P. SHESTAKOV \\ Federal Science Center for Physical Culture and Sport, Moscow, Russian Federation
}

\begin{abstract}
The study intended to compare the training load volume (TrV) distribution of elite Russian (RuXC) and Norwegian (NorXC) cross-country skiers in a one-year macrocycle. Daily TrV of 11 RuXC skiers averaged for the period 2014/15-2017/18. The NorXC skiers' TrV obtained from the study by Sandbakk (2017). RuXC skiers had a lower volume of low-intensity (LIT, below aerobic threshold) and high-intensity (HIT, above anaerobic threshold) endurance training. They used a "pyramidal" model of intensity ratio during the entire macrocycle and did not decrease the volume of moderate-intensity (MIT) endurance training in competition periods (CPs). Conversely, NorXC skiers followed the "pyramidal" model of intensity in the preparation period (PP) but the "polarized" model in CP, significantly reducing the volume of MIT and increasing that of HIT. RuXC skiers increased TrVs more rapidly at the beginning of PPs, achieving TrV peak in June, and then gradually decreased them by March. NorXC skiers increased TrVs gradually by July and then maintained this approximate volume until November. RuXC skiers had peak volumes of LIT and strength training simultaneously in June; NorXC skiers engaged in large amounts of strength training in May and June until reaching maximum endurance loads. RuXC skiers had two "blocks" of strength training; NorXC skiers had three. A comparative analysis of the TrV distributions among the RuXC and NorXC skiers revealed significant similarity. Therefore, they can consider as models of the modern annual periodization of training loads for this kind of sport.
\end{abstract}

Keywords: Sports performance; Endurance training; Strength training; High-Level athlete; Training intensity.

\section{Cite this article as:}

Myakinchenko, E.B., Kriuchkov, A.S., Adodin, N.V., Dikunets, M.A., \& Shestakov, M.P. (2021). One-year periodization of training loads of Russian and Norwegian elite cross-country skiers. Journal of Human Sport and Exercise, 16(3), 701-710. https://doi.org/10.14198/ihse.2021.163.18

Corresponding author. FSBI - Federal Science Center for Physical Culture and Sport, 1/10, Elizavetinskiy Lane, Moscow, 105005, Russian Federation. https://orcid.org/0000-0003-1184-9694

E-mail: eugst@yandex.ru

Submitted for publication February 19, 2020.

Accepted for publication May 04, 2020.

Published July 01, 2021 (in press May 13, 2020).

JOURNAL OF HUMAN SPORT \& EXERCISE ISSN 1988-5202

(c) Faculty of Education. University of Alicante.

doi:10.14198/jhse.2021.163.18 


\section{INTRODUCTION}

In cross-country skiing, from the 1950s to the present, there has been a constant rivalry between two "training schools": that of the Russian and the Scandinavian one. For example, Figure 1 shows that Norway and Russia are still world leaders in cross-country skiing. Therefore, a comparison of the annual training load volumes (TrV) distribution among the athletes from these countries can have a significant scientific and practical interest.

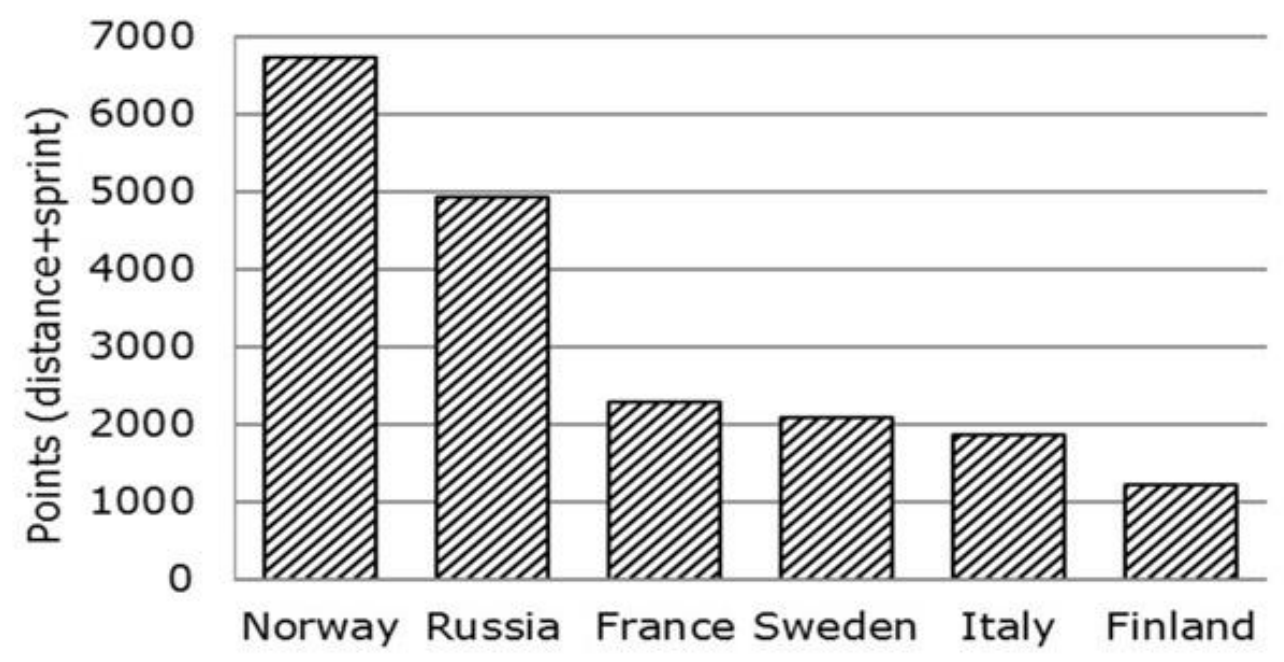

Figure 1. Cross-Country Nations Cup Standings (2019 overall points) for the different nations (International Ski Federation Cup Standings, 2019).

Numerous investigations of the cross-country skiing training process have conducted in recent years. Analyses of training volumes, training intensity ratio and distribution, pre-tapering and tapering phases, tendencies in the development of periodization of adult elite Norwegian cross-country skiers' (NorXC) training processes, and even authentic training plans have presented in a series of studies (Sandbakk, 2017; Sandbakk et al., 2011; Sandbakk and Holmberg, 2014, 2017; Sandbakk and Tønnessen, 2012; Solli et al., 2017, 2019; Tønnessen et al., 2014). However, few studies of cross-country skiers of other countries that trained according to different coaching styles have conducted. In particular, investigations of elite Russian cross-country (RuXC) skiers have not yet published.

At the same time, it can be assumed that identifying the matching parameters of training processes for elite cross-country skiers who use different training systems will allow us to describe the modern characteristics of periodization in this sport. Conversely, an analysis of their differences can stimulate the search for new ways to improve the efficiency of the training process. Therefore, the main objective of this study was to identify differences and similarities in the distribution of TrVs between training programs for elite RuXC and NorXC skiers.

We hypothesized that a study of the characteristics of the distribution of TrVs in the annual cycle of elite cross-country skiers' training would make it possible to determine the aspects of the training process that are important for the theory and practice of training for cross-country skiing. 


\section{MATERIALS AND METHODS}

\section{Participants}

Data for NorXC skiers obtained from Sandbakk's (2017) investigation. Characteristics of elite RuXC skiers selected for this research presented in Table 1. Selection criteria for the RuXC skiers were as follows: 1) all the athletes should have been included in the Russian national team at least twice during the seasons 2014/15-2017/18 (four-year period); 2) all underwent medical check-ups twice a year, and none had experienced trauma or severe illness; thus, everyone was allowed to train; 3) all had been participating actively in the International Ski Federation (FIS) competitions, including the World Championships and the Olympic Games, and they had a FIS ranking of 49 and above. All the athletes signed a contract agreeing to participate in authorized annual training and controlling events during their participation while on a national team and gave informed consent to use their data for scientific purposes on the condition of anonymity. All information collected during the complex monitoring of the Russian athletes' training process by the staff of the Training Centre of the Russian National Teams (Shestakov \& Myakinchenko, 2018). This study conducted following the scientific plan of the FSBI Federal Science Centre for Physical Culture and Sports approved by the Russian Ministry of Sports (Order No. 1034 of 12/14/2018).

Table 1. Mean characteristics of male elite Russian cross-country skiers ( $n=11)$.

\begin{tabular}{lc}
\hline Indicators & Mean value \pm SD \\
\hline Age $(\mathrm{y})$ & $28.1 \pm 3.4$ \\
Weight $(\mathrm{kg})$ & $78.3 \pm 5.1$ \\
Height $(\mathrm{cm})$ & $178.3 \pm 4.8$ \\
$\mathrm{VO}_{2 \max }\left(\mathrm{mL} \cdot \mathrm{min}^{-1} \cdot \mathrm{kg}^{-1}\right)^{*}$ & $81.3 \pm 3.6$ \\
\hline
\end{tabular}

Legend. SD - Standard Deviation; $\mathrm{VO}_{2 \max }-$ Maximal Oxygen Consumption. ${ }^{*} \mathrm{VO}_{2 \max }$ was determined during incremental running with ski poles on a treadmill (Myakinchenko et al., 2019).

\section{Organization of the training process}

Each season, 14 to 16 of the best Russian cross-country skiers invited to train as part of the national team. The athletes divided into three or four teams that trained separately with different coaches during the research period. Each team followed the same training plan. The TrVs of one or two leaders from each team who successfully passed all training periods used to determine the characteristics of the "typical" TrV distribution of the Russian cross-country ski team.

The organization of the training process was as follows. The athletes gathered for joint preparation in various training camps from May to March. Each camp lasted from 11 to 21 days. Additionally, there were periods of "home" training from 6 to 10 days; these included flights covering 2 to 8 time zones. April is traditional vacation time for the athletes when they rest at home or resorts using non-specified supporting training. The pretapering phase was usually from the middle of November to January, while the tapering phase and major competitions took place in February or March.

\section{Methods of monitoring the training}

The daily collection of TrVs was conducted by experienced supervised staff who had been specially trained, given detailed instructions on exercise classification and provided with Excel spreadsheets. The staff accompanied the teams through all the camps from May to March. During the periods of individual training, the athletes recorded and saved their heart rate (HR) data on watches (Polar RX800, Polar Electro Oy, Kempele, Finland), and registered other training information in training diaries. The staff collected that information after the athletes' arrival at each training camp. The TrV data not collected during the recovery 
period in April. However, selective analysis of a number of diaries indicated that the structure of TrVs resembled that of May and that monthly general TrVs were, on average, 25 hours. All the daily training information was sent to the central database by the same staff on a regular basis, where it processed by the researchers.

TrVs of endurance training included all kinds of regular daily endurance training, competitions, warm-ups, cool-downs, and rest periods between the heats and the intervals. A three-zone model and the time-in-zone approach (TIZ) (Sylta et al., 2014) used to quantify the intensity of endurance TrVs. The term "low-intensity endurance training" (LIT, HR < 155-158 beats per minute (bpm), 85-86 \% maximum heart rate (HRmax)) referred to work intensity lower than the aerobic ventilation threshold (AeT). "Moderate-intensity training" (MIT) referred to an intensity between the AeT and anaerobic ventilation threshold (AnT) (HR > 169174 bpm, 91-94 \% HRmax), and "high-intensity training" (HIT) referred to training intensity above AnT. The HRs at AeT and AnT were determined based on regular non-maximum treadmill tests while running with poles using a ramp protocol with speed increments of $0.5 \mathrm{~km} / \mathrm{h}$ per minute at a fixed grade of $10 \%$. Maximal oxygen consumption determined during a three-step test, where athletes ran until exhaustion (Myakinchenko et al., 2019).

To compare TrVs of the RuXC skiers with those of the NorXC skiers presented by Sandbakk (2017), where the hybrid session-goal/time-in-zone (SG/TIZ) approach implemented (Sylta et al., 2014), the correction factors across TIZ and SG/TIZ methods calculated. The conversion factors were as follows: LIT -+1.002 , MIT - - 0.91, and HIT - + 1.32 .

Total strength training in the gym, sprint, and jump time (excluding warm-up and cool-down) recorded. Additionally, the duration of the fitness and stretching sessions calculated.

\section{Statistical analysis}

The data from 29 digital diaries kept by the RuXC skiers were collected, analysed, and averaged monthly. The non-parametric Mann-Whitney U-test was applied based on the abnormally distributed data for differences between the monthly TrVs in the periods of April-November (PP) and December-March (CP). A regression analysis (a linear model or a second-order polynomial) was used separately for every team during the PPs or the entire season to discover a trend in the changes of TrVs. A determination coefficient (R2) calculated, and its significance was determined using a Fisher $F$ criterion. The statistical analyses carried out in IBM STATISTICA for Windows, Version 10.0 (StatSoft, Inc., Tulsa, OK USA). Statistical significance set at a level of .05. To enable a non-statistical comparison of the TrVs of the RuXC skiers with the NorXC skiers, the figures published by Sandbakk (2017) were digitized by visual assessment. Therefore, in this research, we used the approximate values of TrVs of the NorXC skiers.

\section{RESULTS}

The total and monthly averaged TrVs of different intensity and modes in the PPs and CPs for the RuXC and NorXC skiers quantified by a SG/TIZ approach presented in Table 2. The RuXC and NorXC skiers did not differ in regard to total annual training time, but it is highly likely that the NorXC had more LIT, total endurance TrVs, and lower MIT. Among the RuXC, the proportions of LIT, MIT, and HIT were 85.1/8.0/6.8 for the whole year; 85.5/8.4/6.1 in PPs, and 82.3/8.8/8.9 in CPs. Among the NorXC, the proportions were 89.3/5.1/5.6, 89.3/6.4/4.3, and 89.2/2.3/8.5, respectively. In the CPs, in comparison with the PPs, the RuXC had lower monthly volumes $(p<.05)$ of total training time, LIT, and power + strength + sprint training time. However, HIT increased, but MIT and non-specific fitness training did not change. The NorXC skiers noticeably reduced 
MIT in the CP and did not reduce the time of power + strength + sprint training in CP as much as the RuXC skiers. The HIT volume was $\sim 40 \%$ higher for the NorXC skiers. The difference was especially obvious in CPs. Monthly distributions of the total training time, LIT, MIT, and HIT as well as the summary times for all types of strength training in the gym, sprint, and power training for the RuXC and NorXC skiers shown in Figure 2.

Table 2. The averaged total annual training time, monthly training time in preparation periods (MayNovember) and in competition periods (December-March) for elite Russian cross-country skiers in the seasons for 2014/15-2017/18 and the approximate values of elite Norwegian cross-country skiers in the 2014/15 season (Sandbakk, 2017), quantified using the SG/TIZ approach (Sylta et al., 2014), mean \pm SD.

\begin{tabular}{|c|c|c|c|c|}
\hline Training time & Annual & PP (monthly) & $\mathrm{CP}$ (monthly) & CP/PP (\%) \\
\hline \multicolumn{5}{|c|}{ Russian } \\
\hline Total training time $(\mathrm{h})$ & $891 \pm 65$ & $77 \pm 18$ & $68 \pm 4.7$ & $88^{*}$ \\
\hline LIT $(h)$ & $670 \pm 59$ & $58 \pm 10$ & $52 \pm 3.9$ & $86^{*}$ \\
\hline MIT (h:min) & $43 \pm 3.4$ & $3: 28 \pm 2.2$ & $3: 44 \pm 0.7$ & 107 \\
\hline HIT (h:min) & $27 \pm 5.9$ & $2: 00 \pm 1.5$ & $2: 48 \pm 1.7$ & $140^{*}$ \\
\hline Total endurance TrVs (h) & $740 \pm 52$ & $63 \pm 11$ & $59 \pm 5.2$ & 94 \\
\hline Strength + Power + Sprint (h:min) & $77 \pm 8.4$ & $8: 07 \pm 2.8$ & $3: 02 \pm 1.1$ & $36^{*}$ \\
\hline Fitness training (h:min) & $73 \pm 13$ & $6: 00 \pm 2.3$ & $6: 14 \pm 1.0$ & 104 \\
\hline \multicolumn{5}{|c|}{ Norwegian } \\
\hline Total training time $(\mathrm{h})$ & 889 & 81 & 61 & 75 \\
\hline LIT (h) & 715 & 65 & 50 & 77 \\
\hline MIT (h:min) & 41 & $4: 36$ & $1: 18$ & 28 \\
\hline HIT (h:min) & 45 & $3: 06$ & $5: 32$ & 180 \\
\hline Total endurance TrV (h) & 801 & 73 & 56 & 77 \\
\hline Strength + Power + Sprint (h:min) & 77 & $8: 33$ & $4: 34$ & 53 \\
\hline
\end{tabular}

Legend. LIT - Low-Intensity Training (HR < 155-158 bpm, 85-86 \% HRmax); MIT - Moderate-Intensity Training; HIT - HighIntensity Training (HR > 169-174 bpm, 91-94 \% HRmax); TrV - Training Volume; PP - Preparation Period; CP - Competition Period. The "Total endurance TrV" is the sum of LIT, MIT, and HIT. "significant difference between PPs and CPs $(p<.05)$.

As shown in figures $2 \mathrm{~A}, 2 \mathrm{~B}$, and $2 \mathrm{C}$, the Russian and Norwegian teams had the largest numbers of total training hours, LIT, and MIT in June and July, respectively. Then both teams constantly (for all, $p<.022$ ) reduced TrVs until March. However, the MIT volume for the RuXC skiers remained relatively stable from June to February (R2 = .55), while for the NorXC skiers, the degree of decrease was much higher (R2 = .94). HIT volume did not fundamentally change from June to October among the RuXC skiers and from July to November among the NorXC skiers $(p=.21$ and $p=.44$, respectively) but increased from May until March across teams $(p<.01)$. At the same time, the NorXC skiers sharply reduced volumes of MIT and increased HIT in December. The strength/power/sprint TrVs had peak volumes in June and have grouped in concentrated "blocks" The first was in June/July, and the second in September/October in both teams. However, in January, the NorXC skiers applied another "block" of strength training, while the RuXC skiers steadily reduced that type of training load from November to March $(p<.01)$. In April and May, the NorXC skiers did not decrease their TrVs as much as the Russian athletes. The difference especially expressed in the volume of strength training. 


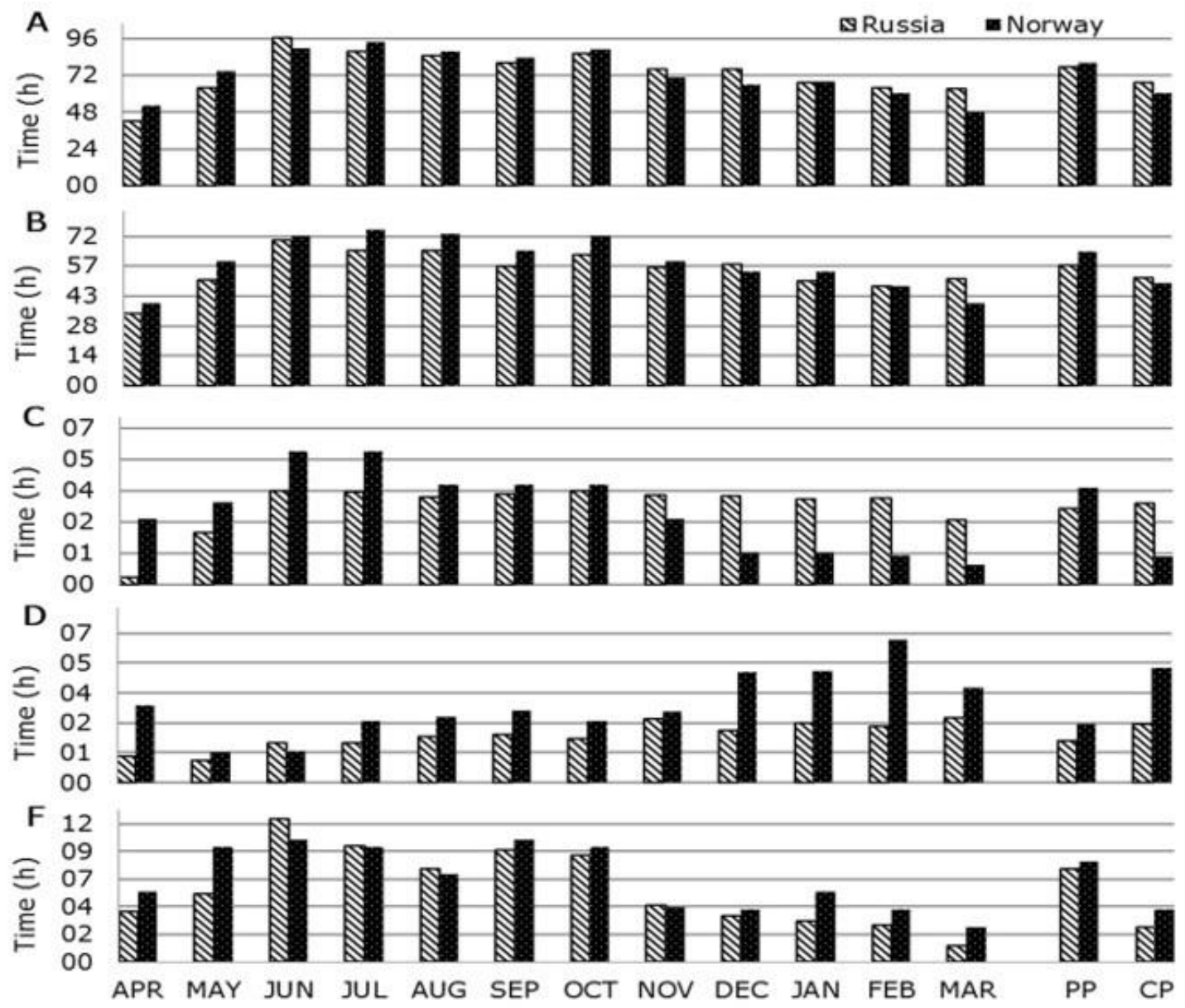

Figure 2. (A) - Monthly distribution of total training hours; $(B)$ - low-intensity endurance training $(H R<155$ 158 bpm, 85-86 \% HRmax); (C) - moderate-intensity training (HR between aerobic and anaerobic thresholds); (D) - high-intensity training (HR > 169-174 bpm, 91-94 \% HRmax); (F) - strength+power+sprint training time for the elite Russian and Norwegian cross-country skiers. PP - average in the preparation periods, CP - average in the competition periods. Data for the Norwegian skiers was modified from Sandbakk, 2017.

\section{DISCUSSION}

In this study, the distribution of TrVs for the elite RuXC and NorXC skiers compared. The data allow us to describe the main matching characteristics and differences in the training processes of these highly competitive teams.

For the leading athletes on the Russian team, the average annual training time for four years was 891 hours (796-991 hours). For the Norwegian winners of the 2015 World Cup, the value was $~ 889$ hours. Thus, this index is almost equal for both teams. However, TrVs of the RuXC skiers included all the training loads collected daily on 39 indexes and included non-specific fitness training. At the same time, in personal communication, Øyvind B. Sandbakk reported that some part of the non-specific training (for example, stretching) might not have included in the NorXC skiers' TrVs. If so, then the actual annual training time for the RuXC skiers would be less, by $70-75$ hours, than that of the NorXC skiers. One of the explanations 
could be Russia's huge geographic size and its "centralized system" of training camps: at the result, some of the skiers lost at least two training days every month and experienced additional non-specific "stress" due to regular trips to training locations. Nevertheless, it could also be that Russian coaches consciously compensated for the lower LIT volume by using toning up circular strength training, games, swimming, etc., which included in the amount of "non-specific fitness training" for the Russian team.

An analysis of monthly TrVs for both teams (see Figure 2) suggests that the prevailing tendencies in TrV distributions are very similar and could reflect the characteristics of modern periodization for elite athletes in cross-country skiing. In particular, minimal TrVs observed in the months following the end of the competition periods. The NorXC skiers had it in March and the RuXC skiers in April (Russian national championships usually held at the end of March). The total amount of TrVs in this month was lower among RuXC skiers ( 43 $\%)$ than NorXC ( $\sim 52 \%)$. That means that the RuXC skiers have reduced their training load more or had a longer vacation in April.

In order to reach maximum TrVs gradually, the stage of preliminary loads followed after the "rest." During that period, the NorXC skiers used a smoother gradient of the TrVs' increase. They had large volumes in April and May, and their TrVs peaked for four months (April-July), while the RuXC skiers reached peak endurance and strength-training volumes for two months (May and June). Thus, the maximum TrVs reached in June and July in both teams. After that, a phenomenon that L. Matveev (1964) called "the principle of transition from quantity to quality" manifested itself. Namely, the volumes of LIT, MIT, and non-specific strength training gradually decreased, while at the same time, the intensity and - apparently - the specificity of the training load increased due to the HIT increasing; that happened from June until the time of the main competitions in February or March.

The difference between the two teams in TrVs organization was that the NorXC skiers had reasonably stable monthly volumes of total training time, LIT, and strength training from June till October (R2 = .17-.35). However, these decreased considerably in November. At the same time, the RuXC skiers decreased TrVs steadily from June to March.

There was no significant difference among teams in LIT volumes for the entire season. It should only be noted that the NorXC skiers had a bit more LIT in PP and lower in CP. However, the organization of MIT and HIT differed significantly. For example, among the NorXC skiers, a reciprocal change in MIT and HIT is observed. In this team, MIT reached maximal volumes in June and July, and then gradually decreased to a minimum at the beginning of the $\mathrm{CP}$.

Conversely, HIT progressively increased up to November and then sharply enlarged in the CP. As a result, for the NorXC skiers, the "pyramidal" model of training intensity ratio in the PP was replaced by the "polarized" model in the CP. At the same time, the RuXC skiers had almost constant monthly values for MIT and more smoothed dynamics for HIT volumes and used the "pyramidal" model throughout the season. Generally, the NorXC used significantly more HIT than the Russian team, especially in the pre-peaking and peaking phases (December-February).

From our point of view, it is worth noting a notable difference in the strength/power/sprint training organization. First, the NorXC skiers began to use considerable volumes of such type of training loads in April and May when the amount of endurance training was not yet as large. This means that they used the principles of training-load organization, which previously had called "advancing the development of the neuromuscular system in endurance kinds of sport at the beginning of the season" and "time separation of loads with different 
training effects" (Verkhoshansky, 1988). At the same time, the RuXC skiers used the largest volumes of endurance and strength training simultaneously - in June and July. Second, both teams used block periodization of strength training (Verkhoshansky, 1970) in PPs. The first one was at the beginning of the year's macrocycle and the second in September/October. However, the NorXC skiers applied another "miniblock" of strength training in January, just before the peaking phase. While the RuXC skiers steadily reduced that type of training load in CP from November to March. Third, the RuXC skiers had a lower volume of strength training, especially in the $\mathrm{CP}$.

To conclude, the distribution of TrVs among elite RuXC and NorXC skiers had many similar features. This can reflect the parameters of modern annual training-load periodization for elite athletes in this sport. The main differences between the teams were as follows: A) the NorXC skier had more endurance TrVs due to LIT and HIT; B) they more smoothly increased training loads at the beginning of the season, while the RuXC skiers had already used maximum volumes of loads in June: $C$ ) the NorXC skiers had MIT peaks in June and July and HIT in December-February, i.e., the use of the "pyramida" model in PP and the "polarized" model in CP observed; the RuXC skiers used a "polarized" model throughout the season; D) the NorXC skiers started the training season with relatively large volumes of strength loads (April-June) and separated it from the peak of endurance training (July).

\section{CONCLUSION}

The purpose of cross-country ski training is to provide the highest level of specific physiological capacities, motor abilities, and the maximal efficiency of the movement system. This allows athletes to achieve competitive speeds at the main races. Besides adequate training exercises and optimal methods, the most important conditions for achieving the highest performance level in due time are the rational distribution of training loads and the effective relationship of their modes during a one-year macrocycle (i.e., their annual periodization). This article presents and compares the annual periodization of the training loads of the two best cross-country teams in the world. Therefore, such a distribution of training loads can be regarded as a model that provides the best adaptation of the skiers, reflects the current level of development of sports science, and practical experience in the field of training methods for elite athletes. In addition, there cannot be a complete coincidence of training models among athletes from different training schools. This is especially true for Russia since the development of sports training theory and methodology in this country is different from those in other countries. Therefore, a comparison of the Norwegian and Russian models is of particular interest to the practice of athlete training.

\section{AUTHOR CONTRIBUTIONS}

M. Shestakov supervised the project and findings of this work. E. Myakinchenko took the lead in writing the manuscript. A. Kriuchkov developed the theory and performed the computations. N. Adodin and M. Dikunets collected, systematized the data and edited the text. All authors discussed the results and contributed to the final manuscript.

\section{SUPPORTING AGENCIES}

No funding agencies were reported by the authors. 


\section{DISCLOSURE STATEMENT}

No potential conflict of interest was reported by the authors.

\section{ACKNOWLEDGEMENTS}

The authors would like to express their appreciation to the supervised coaches of the Russian national teams, namely, Max Volkov, Egor Sorin, and Natali Novikova for their skilled work on data collection for this study, and all the athletes of the Russian National XC ski teams and their coaches for their active participation in the study.

\section{REFERENCES}

International Ski Federation (FIS). Cup Standings. (2019). Retrieved from: https://www.fisski.com/DB/cross-country/cup-standings.html?sectorcode=CC\&seasoncode=2019\&cupcode=NCWC\&disciplinecode=ALL\&gendercode=M\&nationcode=

Matveev, L. P. (1964). Problema periodisatzii sportivnoy trenirovki [A problem of periodization of sports training]. Moscow, Union of Soviet Socialist Republics: Fizkultura I Sport.

Myakinchenko, E., Binghong, G., \& Shestakov, M. (2019). Comparative analysis of the use of moderatealtitude training by top Russian and Chinese athletes. Polish Journal of Sport and Tourism, 26(1), 20-27. https://doi.org/10.2478/pjst-2019-0004

Sandbakk, $\varnothing$. (2017). The evolution of champion cross-country-skier training: From lumberjacks to professional athletes. International Journal of Sports Physiology and Performance, 12(2), 254-259. https://doi.org/10.1123/ijspp.2016-0816

Sandbakk, Ø., Ettema, G., Leirdal, S., Jakobsen, V., \& Holmberg, H. C. (2011). Analysis of a sprint ski race and associated laboratory determinants of world-class performance. European Journal of Applied Physiology, 111(6), 947-957. https://doi.org/10.1007/s00421-010-1719-9

Sandbakk, $\varnothing$., \& Holmberg, H. C. (2014). A reappraisal of success factors for Olympic cross-country skiing. International Journal of Sports Physiology and Performance, 9(1), 117-121. https://doi.org/10.1123/ijspp.2013-0373

Sandbakk, Ø., \& Holmberg, H. C. (2017). Physiological capacity and training routines of elite crosscountry skiers: Approaching the upper limits of human endurance. International Journal of Sports Physiology and Performance, 12(8), 1003-1011. https://doi.org/10.1123/ijspp.2016-0749

Sandbakk, Ø., \& Tønnessen, E. (2012). Den norske langrennsboka (The Norwegian cross-country book). Oslo, Norway: H. Aschehoug \& Co. (W. Nygaard).

Shestakov, M., \& Myakinchenko, E. (2018). Control and management in athletic training of the Russian national teams. Journal of Sports Science, 6(2), 118-128. https://doi.org/10.17265/23327839/2018.02.007

Solli, G. S., Tønnessen, E., \& Sandbakk, Ø. (2017). The training characteristics of the world's most successful female cross-country skier. Frontiers in Physiology, 8, 1069. https://doi.org/10.3389/fphys.2017.01069

Solli, G. S., Tønnessen, E., \& Sandbakk, Ø. (2019). Block vs. traditional periodization of HIT: Two different paths to success for the world's best cross-country skier. Frontiers in Physiology, 10, 375. https://doi.org/10.3389/fphys.2019.00375

Sylta, O., Tønnessen, E., \& Seiler, S. (2014). From heart-rate data to training quantification: A comparison of 3 methods of training-intensity analysis. International Journal of Sports Physiology and Performance, 9(1), 100-107. https://doi.org/10.1123/ijspp.2013-0298 
Tønnessen, E., Sylta, O., Haugen, T. A., Hem, E., Svendsen, I. S., \& Seiler, S. (2014). The road to gold: Training and peaking characteristics in the year prior to a gold medal endurance performance. PLoS One, 9(7), e101796. https://doi.org/10.1371/journal.pone.0101796

Verkhoshansky, Y. V. (1970). Osnovi spetzialnoy silovoy podgotovki v sporte JFundamentals of special strength-training in sport]. Moscow, Union of Soviet Socialist Republics: Fizkultura I Sport.

Verkhoshansky, Y. V. (1988). Osnovi spetsialnoy fizitceskoy podgotovki sportsmenov [Fundamentals of special physical training in athletes]. Moscow, Union of Soviet Socialist Republics: Fizkultura I Sport.

\section{(c) $(1) \ominus$}

This work is licensed under a Attribution-NonCommercial-NoDerivatives 4.0 International (CC BY-NC-ND 4.0). 